\title{
FORMACIÓN DOCENTE Y SABERES LOCALES: UNA MIRADA DESDE LA RURALIDAD. VENEZUELA
}

Jesús Núñezl

\section{Resumen}

La formación docente en los países latinoamericanos se encuentra inmersa en una serie de complejidades y contrariedades. Los educadores, como formadores de una sociedad, reproducen sin pausa e inconscientemente, en su mayoría, los saberes modemos deudores de la cultura occidental. Sin embargo, los contextos de la acción docente exhiben una gran riqueza acumulada en las sabidurías de los pueblos, que son ignoradas en los programas escolares formales. Se propone en esta ponencia, la necesidad urgente de otorgar mayor pertinencia cultural y social a los saberes que se enseñan desde la escuela. Para ello, son claves dos aspectos: otorgar un estatus académico a los saberes locales (potenciados por las epistemes emergentes ante la crisis de la modemidad) y establecer un diálogo intercultural entre el saber local y el universal, para aprovechar sus fortalezas, asumiendo una postura de sinergismo de los saberes humanos.
Palabras claves: saberes locales y modemos, educación, ruralidad.

\section{Abstract}

Teacher's education in LatinAmerican countries is surrounded by complexity and contradiction. Teachers reproduce continuously and mostly unconsciously modem knowledge inherited of westem culture. However, the context of educational activity exhibits great richness, which has been accumulated from the wisdom of people ignored in the curricula of formal educational programs. The article proposes the urgent need of giving social and cultural relevance to the knowledge that is being taught in schools. Two key aspects are proposed: first, giving academic status to local knowledge, powered by the emerging episteme of the crisis of modemity; and second, the necessity of establishing an intercultural dialog between local and universal knowledge, in order to take advantage of its strengths,

1 Acadèmico venezolano, perteneciente al Núcleo de Investigación EDUCA, Línea de Investigación: Campesinos, Educación y Ruralidad (CER), UPEL-IPRGR. Ha participado como ponente en la Jomada de Educación Integral del Instituto Pedagógico Rural Gervasio Rubio. jesus57@cantv.net

Recibido: 30 de octubre-2006 - Aprobado: I5 de noviembre-2006 
assuming a synergic posture about human knowledge.
Keywords: local and modern knowledge, education, rural contexts.

\section{Desarrollo}

$\mathrm{H}$ ablar de formación docente en Venezuela, y especialmente en nuestros espacios andinos, supone realizar una abstracción de los procesos inherentes a ella, para tomar la distancia necesaria y cuestionar la misión propia de las universidades que administran programas de formación docente. Son muchos los ángulos desde los cuales se pueden visualizar las características que perfilan al docente, por lo que, sin pecar de ambiciosos, pretendemos realizar un primer acercamiento para interpretar la pertinencia en la formación de los educadores desde la óptica de las culturas locales de los pueblos.

En el desarrollo de esta discusión, trataremos, en lo posible, de dar respuestas a las siguientes interrogantes: ¿cómo se educan los docentes que laboran en las escuelas rurales?; ¿quiénes educan a los niños campesinos y a las niñas campesinas?; ¿qué enseñan en las escuelas rurales?; ¿qué lugar ocupan los saberes locales en la educación rural? y ¿cómo lograr darles estatus académico a los saberes locales? Haremos el mayor énfasis de la disertación en las dos últimas preguntas.

Desde el punto de las políticas educativas, los y las docentes, licenciados por el Estado venezolano para ejercer cargos de maestros en las escuelas públicas rurales, egresan de instituciones de educación superior, tanto autónomas como experimentales, en las diferentes áreas del conocimiento, preferiblemente como especialistas en Educación Básica Integral o Rural; sin embargo, como veremos en párrafos posteriores, existe una disonancia entre la especialidad en la formación docente y las especificidades socioculturales de los sujetos a educar.

Durante el proceso de formación académica, el futuro docente es educado con base en diseños curriculares que reproducen la cultura occidental moderna referidos a la enseñanza de asignaturas bajo marcos referenciales disciplinarios, particularizados y atomizados, que tienen como norte detectar y solucionar problemas parciales (Ishizawa, citado por PRATEC, 1998) y que pretenden seguir colonizando las mentes y las acciones de los habitantes de las localidades intervenidas.

En la formación de los docentes para reproducir social y culturalmente los modelos hegemónicos (Giroux, 1995), coexiste una serie de estilos de 
pensamiento (Padrón, 1994) de los profesores universitarios que marcan y complejizan la personalidad académica del educando ante la presencia de una selva de estilos epistemológicos, que a la larga son causantes de profundas contradicciones entre lo que diseña el Estado venezolano como políticas educativas, lo que se enseña en las universidades y lo que esperan las comunidades locales (Núñez, 2000).

La formación diferenciada de profesores por especialistas y superespecialistas, egresados de las diversas ramas del saber modemo, dificulta las posibilidades de comprender e interpretar las cosmovisiones holísticas de las comunidades locales por el docente de educación básica. Es bien difícil, entonces, desde los estancos de los conocimientos compartimentados de la ciencia modema, aprovechar la riqueza de las sabidurías locales, las cuales no pueden ser medidas, validadas, objetivadas y generalizadas como lo exige la ciencia positivista, todavía hegemónica en el pensar y actuar de la ciencia.

En síntesis, los docentes venezolanos son educados dentro de un modelo impuesto a nuestras especificidades socioculturales y ambientales para reproducir los proyectos emancipadores, renovadores, democratizadores y expansivos (García Canclini, 1989) propios de la modemidad, que han abrazado a nuestros territorios y culturas y que, con mayor ferocidad en los últimos 20 años, ha abierto los caminos para la globalización, es decir, la homogeneización de lo heterogéneo y biodiverso.

Este cuestionamiento a los saberes que se enseñan para ser enseñados a los pueblos en la formación de los ciudadanos modemos es posible que no sea comprendido o aceptado por todos. Eso es entendible, puesto que en el proyecto emancipador y expansivo, estos saberes fueron sistemática y continuamente naturalizados (Lander, 1998) en las formas y mundos de vida de los países dominados. Según González Stephan (1992, citado por Lander), existieron tres prácticas disciplinarias (las constituciones nacionales, los manuales de urbanidad y las gramáticas de la lengua) que desde el siglo XIX fueron impulsadas desde los Estados-Nación y reproducidas en los programas educativos -la escuela-, el arte y la literatura para formar a los ciudadanos latinoamericanos. Para ilustrar estas aseveraciones, vasta con recordar el famoso Manual de Carreño, publicado en el año 1854, y de frecuente alusión aún entre nosotros, que buscaba domesticar nuestros instintos salvajes a través del teatro de la etiqueta. Hoy en día, desde la vida cotidiana hasta la más intelectual, los instintos considerados bárbaros son repudiados por las personas cultas y son signos de marginalidad, falta de educación, propios de clases sociales culturalmente deprimidas. 
Inmersos en esta dimensión del saber, los docentes licenciados por las universidades nacionales están preparados para cumplir con su misión profesional de contribuir al desarrollo del país, proceso que después del acto de grado académico les puede deparar muchas sorpresas, alegrías o decepciones, ya que "la vida es jacarandosa, a veces negra, a veces rosa...", como dice una canción de moda. En Venezuela, la inserción de los docentes recién graduados al campo laboral representa uno de los procesos más traumáticos por los que debe pasar un profesional. Tanto en las llamadas IV y V Repúblicas, los denominados concursos de ingreso de personal docente se constituyen en focos que potencian la corrupción y el manejo de espacios de poder. Es así como, producto de estos procesos de selección, ingresan como docentes a las escuelas rurales profesionales formados para diversas modalidades y niveles del sistema educativo. En el país, las políticas educativas no las establece el Estado sino los gobiemos (Cortés, 2002) y menos aún para los grupos campesinos e indígenas, contextos socioculturales donde se puede afirmar que no existe educación rural, sólo una educación urbana en escuelas rurales (Núñez, 2000).

Se tiene, entonces, que en las desparramadas escuelas rurales, que abarcan una amplia gama de formas arquitectónicas, que van desde tristes ranchos de tabla sin los servicios mínimos indispensables, pasando por caserones antiguos hasta los "modemos R1 y R2", donde se educan a los niños en los dificiles multigrados o en aulas para grados diferenciados, pero que en todo caso no superan los primeros 6 años de la educación básica rural. Coexisten en ellas, en perpleja dificultad existencial y profesional también, una amplia variedad de docentes. Hacen vida en las escuelas rurales: bachilleres, docentes no graduados, Licenciados y Profesores en Educación Integral, Profesores en Educación Rural, Especialistas de las más diversas áreas de la Tercera Etapa, Media y Profesional de la Educación, entre otros. Es decir, en las complejidades de los entomos rurales también se albergan las complejidades de sus actores educativos.

Una vez insertados en las comunidades rurales, el quehacer del docente se centra, principalmente, en atender las normativas y programas curriculares emanados de las instancias educativas superiores, por lo que en el tiempo escolar, se va replicando dentro de la escuela un saber generalizado y poco pertinente con su entorno y que pretende, a costa de mutar los mundos de vida propios de las culturas locales, formar un modelo de hombre o mujer competitivo e individual, característico del capitalismo. Es aquí donde la normalización del saber moderno instituido cobra altos niveles de eficiencia, pues 
es tan profundo el arraigo en el ser que educa, que es dificil tomar conciencia del rol asumido como colonizador de mentes y acciones en el educando. No importa que la acción educativa esté presente en el campo o en la ciudad, en el páramo, montaña o llano, en todos estos espacios temporo-ambientales, multidiversos y complejos, la escuela repite, reproduce e inserta sin pausa una cultura globalizada a espaldas de las realidades inmediatas de sus actores naturales.

Pero, ¿es que hay algo que ha olvidado la escuela, que es de notable riqueza en las comunidades locales? Indudablemente que sí, pues con el afán de estar en la misma ola donde se mueven los conceptos y teorías universales del conocimiento científico -la punta tecnológica-, hemos obviado nada más y nada menos que nuestras propias esencias culturales. En otras palabras, es como cuidar solo el cuerpo -la apariencia- y dejar de lado el alma -la esencia- de nuestros pueblos. Se asumen programas y modelos educativos y se esmeran porque, operativamente, funcionen bien en el sistema educativo local; mas desde sus entrañas, las voces de nuestros mayores gritan dentro de una burbuja de silencio y olvido. Son muchas las preguntas que empujan por salir: ¿se le ha preguntado al padre campesino o la madre campesina qué quiere que sus hijos aprendan?, ¿se han estudiado los procesos cotidianos mediante los cuales aprenden los grupos campesinos?, ¿cómo enseñar desde una racionalidad moderna parcializada a las culturas campesinas con racionalidad holística?, ¿cuál es el valor otorgado por el docente educador a los saberes locales presentes fuera de los linderos de la escuela?

Algunos de ustedes pensarán que las consideraciones enunciadas son meras especulaciones y, por lo tanto, estos saberes no tienen validez científica. Pues, ese ha sido el principal problema de la ciencia moderna -actualmente en crisis- que al desplazar el dogma de la fe del período Escolástico colocó la razón como su nuevo Dios y con ello deshumanizó la vida, eliminó al ser humano y sus creencias de toda apreciación razonable. En consecuencia, todo aquello que no es científico es pura especulación, se arroja a la metafísica.

Alejandro Moreno (2002) considera que el ser humano habita en dos mundos: el moderno y el popular, y Córdoba (2002) en concordancia con Morin (1999) son partidarios de la coexistencia al mismo tiempo del hombre en esos dos mundos. Es decir, hacemos vida en ambos mundos aunque a veces solo creemos estar en uno de ellos. Kusch (1975, citado por Castro, 1996), por su parte, afirma que el sujeto latinoamericano posee dos subcapas culturales, una epidermis delgada producto de la imposición y una dermis 
profunda por la riqueza de las culturas autóctonas. Este mestizaje cultural es lo que García Canclini (1989) denomina culturas híbridas.

En la vida profesional, hacemos un culto supremo al mundo moderno aunque estemos inmersos en un rico entramado del tejido social donde reina el saber popular, local, cotidiano, común, campesino, entre otros. En él se acumulan y se re-crean dinámica y transgeneracionalmente unas estructuras de significado que han garantizado la supervivencia del ser humano a lo largo del tiempo. Es un corpus de saberes teóricos y prácticos (Agruco, 1998, citado por Arratia, 1999), formado a través de las adquisiciones técnicas de los grupos sociales en miles de años de evolución (Carbonell y Sala, 2000) y que son expresadas mediante símbolos, representaciones, conceptos, prácticas y modos de vida cotidianos, que para ser asumido por el actor de cada generación, debe ser ambientalmente sostenible, socialmente pertinente, culturalmente compatible y humanamente necesario.

Los saberes locales, de acuerdo con estudios realizados por el Programa Andino de Tecnologías Campesinas (PRATEC) en el Perú (1998), siguen un proceso inductivo en su construcción, similar al conocimiento científico positivista, pero que a diferencia de este, no parcializa la realidad para generalizarla por sus regularidades, sino que forma estructuras fuertemente imbricadas por interacciones de los elementos contextuales, naturales, humanos, sociales y culturales. Allí es donde se da la gran diferencia entre estas dos formas de saberes que, en suma, representan dos cosmovisiones divergentes sobre la forma de ver el mundo.

En el ámbito educativo, estas dos cosmovisiones se reproducen en formas opuestas: la moderna es enseñada por programas de estudio escolarizados y operacionalizados a través de la escuela en mediación obligada del lenguaje escrito, y la local es enseñada y re-creada en el diario vivir con la familia y con los vecinos mediante el uso exclusivo de la oralidad y la memoria individual y colectiva.

En la escuela, tanto la urbana como la rural, los saberes locales tienen poca o ninguna importancia al ser desechados o sólo no considerados relevantes para enseñar a la persona actual. Parra de Chópite (2002) asevera que a pesar que el Currículo Básico Nacional (1996) ha dejado un espacio (30\%) para contextualizar los programas educativos a las especificidades culturales de las comunidades, la educación se encuentra en deuda con las acciones para revalorizar las culturas locales.

Conceder estatus académico a la sabiduría popular pasa, a nuestro modo de ver, por dos procesos concatenados, quizás irreverentes para el 
ciudadano moderno. En primer lugar, el docente debe ser formado para un diálogo intercultural (Delgado, 1998) basado en el mutuo respeto de saberes y el aprovechamiento sinérgico de las fortalezas de cada uno de los sistemas de pensamiento. Esta potenciación del saber emergente podría otorgar mayor pertinencia social y cultural a lo enseñado desde la escuela. El segundo proceso, de hecho el más difícil, es asumir una postura epistemológica de desaprender para aprender. Desaprender la ciencia moderna con su visión atomizada de la realidad para comprender e interpretar las cosmovisiones integrales de las comunidades locales dentro de las cuales no hacen vida una racionalidad única, sino múltiples y entramadas formas de construir y hacer la vida.

Lo planteado en todo este discurso pretende mover el piso de las ideas tradicionales sobre las cuales estamos afincados por y desde la educación recibida como sujetos de la modernidad. Supone la urgencia, necesidad e importancia de apostar a la construcción de un nuevo orden civilizatorio desde nuestras fortalezas y debilidades, desde el otro olvidado y dominado, que en esta crisis de la modernidad y de entrecruzamientos de tiempos, debe hacer oír las voces de la capa cultural profunda y mestiza de nuestros pueblos, quienes hace más de 500 años tuvieron un proyecto de hombre amerindio, el cual fue abruptamente interrumpido por la violencia física y epistémica (Bohórquez, 1988) de la cultura occidental. 


\section{REFERENCIAS}

Arratia, O. (1999). Cassette-foro y la revalorización del saber campesino. Universidad Mayor de San Andrés, Facultad de Ciencias Sociales. Tesis de pregrado no publicada para optar al título de comunicación social, La Paz, Bolivia.

Bohórquez, C. (1988). Violencia, filosofia e historia en América Latina en ¿Hacia donde va América Latina? Gastón Parra Luzardo (coordinador, 1996). Venezuela: Editorial LUZ.

Castro, S. (1996). Critica de la razón latinoamericana. España: Editorial Puvill Libros, S. A.

Castro Gómez, S. (2000). Ciencias sociales, violencia epistémica y el problema de la invención del otro en la colonialidad del saber: eurocentrismo y ciencias sociales. Perspectivas latinoamericanas. Venezuela: Edgardo Lander-Editor, edición FACES/UCV-UNESCO.

Córdoba. (2002). Defensa de Tesis Doctoral en VI jornadas institucionales de investigación. Instituto Pedagógico Rural Gervasio Rubio.

Cortés, I. (2002). Educación, globalización y universidad. Conversatorio en el Núcleo de Investigación en Educación, Cultura y Cambio (EDUCA). Instituto Pedagógico Rural Gervasio Rubio, Estado Táchira.

Delgado, F. (1998). El conocimiento científico y el saber local en un diálogo intercultural para el fortalecimiento de la gestión municipal y el desarrollo sostenible. AGRUCO de agricultura $\mathrm{N}^{\circ} 30$. Recuperado en: http://www.agruco. org/articulo6. 1 html, Bolivia.

Garcia C. (1989). Culturas hibridas: estrategias para entrar y salir de la modernidad. México: Editorial Grijalbo.

Giroux, H. (1995). Teoria y resistencia en educación. España: Editorial Siglo XXI. Moreno, A. (2002). Defensa de Tesis Doctoral en VI jornadas institucionales de investigación. Instituto Pedagógico Rural Gervasio Rubio.

Morin, E. (1999). Los siete saberes necesarios para la educación del futuro. Colombia: UNESCO, Editorial Cooperativa del Magisterio.

Núñez, J. (2000). Disonancias epistemológicas en la educación rural venezolana (una aproximación a su interpretación). Mimeo. Doctorado en Educación, Instituto Pedagógico Rural Gervasio Rubio.

Padrón, J. (1994). Modelo de las correspondencias entre enfoques epistemológicos y estilos de pensamiento. Mimeo. Universidad Simón Rodríguez, 1-13.

Parra de Chópite, B. 2002. Foro sobreformación docente. VI jornadas institucionales de investigación. Instituto Pedagógico Rural Gervasio Rubio.

PRATEC (1998). La regeneración de saberes en los andes. Lima, Perú: Gráfica Bellido. 